\title{
La importancia de la nutrición y los estilos de vida saludables en la infancia y adolescencia
}

\section{The importance of nutrition and healthy lifestyles in childhood and adolescence}

\author{
N. Martín-Calvo ${ }^{1,2,3}$, L. Moreno-Galarraga ${ }^{3,4}$, M. Bes-Rastrollo ${ }^{1,2,3}$
}

\section{Sr. Director:}

Habiendo leído con atención la editorial de la Dra. Marti y el Dr. Martínez acerca de la necesidad imperiosa de actuar de forma inmediata en la alimentación del adolescente $^{1}$, queremos mostrar nuestro máximo acuerdo con los autores cuando urgen a frenar el avance de la obesidad mediante la adquisición de hábitos de vida saludable en edades tempranas de la vida.

A nivel mundial, según datos de la OMS, en 2013 había 42 millones de niños/as menores de 5 años con sobrepeso u obesidad, 10 millones más que en $1990^{2}$. Según el estudio ALADINO 2013³, en España, la prevalencia de sobrepeso y obesidad en los niños/as de entre 7 y 8 años es del $24,6 \%$ y $18,4 \%$ respectivamente, ligeramente inferior a la hallada en 2011. Si bien la estabilidad de las cifras es un dato esperanzador, España sigue siendo a día de hoy uno de los países con mayor prevalencia de sobrepeso y obesidad infantil.

Se ha sugerido que la epidemia de obesidad que vivimos en la actualidad podría ser el resultado de una interacción entre diversas variantes genéticas y el ambiente obesogénico que caracteriza a los países desarrollados ${ }^{4}$. Un trabajo recientemente publicado, que recoge los hábitos dietéticos de más de 1000 adolescentes andaluces, pone de manifiesto, una vez más, la tendencia a la occidentalización de la dieta, con una mayor frecuentación de establecimientos de comida rápida, en detrimento de patrones más saludables, como el patrón de dieta mediterráneo ${ }^{5}$. Las conclusiones de estos autores se unen a las de otros trabajos que ya habían sugerido que los hábitos poco saludables tienden a agruparse, de manera que existe una asociación entre el consumo de alimentos de escaso valor nutricional, una reducción de la actividad física y un mayor tiempo de ocio pasivo ${ }^{6}$.

En este escenario, consideramos que el fomento de medidas que promuevan la adquisición de hábitos dietéticos y estilos de vida saludables ente los niños/as y adolescentes debe ser una prioridad de Salud Pública. Bajo esta premisa, en enero de 2015 iniciamos el proyecto SENDO (Seguimiento de Escolares Navarros para un Desarrollo

An. Sist. Sanit. Navar. 2015; 38 (3): $461-462$

1. Departamento de Medicina Preventiva y Salud Pública. Universidad de Navarra, Pamplona.

2. CIBER Fisiopatología de la Obesidad y Nutrición (CIBERobn), Instituto de Salud Carlos III, Madrid.

3. Instituto de Investigación Sanitaria de Navarra (IdiSNA).

4. Servicio de Pediatría. Complejo Hospitalario de Navarra, Pamplona.

\section{Correspondencia:}

Nerea Martín-Calvo

Departamento de Medicina Preventiva y Salud

Pública

Universidad de Navarra

Campus Universitario plantas 1-5

31008 Pamplona

E-mail: nmartincalvo@unav.es 
Óptimo), un proyecto piloto para el que reclutamos más de 300 niños/as de entre 4 y 6 años de Navarra.

La principal fuente de información en nuestro estudio la representan los padres de los participantes, y el principal método de recogida de información son los cuestionarios en papel, siguiendo el modelo de otros estudios epidemiológicos llevados a cabo con éxito en niños/as y adolescentes? En mayo de 2015, los más de 300 participantes recibieron un cuestionario dividido en cuatro grandes apartados: datos sociodemográficos (donde se recogen además antecedentes personales y familiares de interés, así como información retrospectiva acerca del periodo perinatal y los primeros años de vida), cuestionario de frecuencia de consumo de alimentos, preguntas acerca de sus hábitos alimentarios y preguntas sobre las actividades física y de ocio.

El proyecto SENDO nace con el objetivo principal de estudiar los hábitos dietéticos y los estilos de vida de los niños/as en edad escolar, para valorar una posible asociación entre estos factores y la obesidad en primer término, pero también con otros desórdenes prevalentes durante el crecimiento y desarrollo (síndrome metabólico, asma, alergias...). Dentro del estudio de los hábitos dietéticos, queremos centrar nuestra atención en el patrón de dieta mediterránea, ya que si bien existen numerosas publicaciones que sugieren un efecto protector del patrón de dieta mediterránea sobre la salud del adulto ${ }^{8}$, todavía son pocos los estudios dirigidos a analizar el efecto de este patrón dietético sobre la salud del niño/a y del adolescente.

Confiamos que el proyecto SENDO sirva de base para la creación de una cohorte mayor formada por niños/as y adolescentes de Navarra; una cohorte abierta y multipropósito de referencia en España, que permita el abordaje de un problema tan plural como es la obesidad infantil desde una perspectiva multidisciplinar, con el compromiso de todos los profesionales implicados en el cuidado, la prevención y la promoción de la salud de los niños/as.

\section{BIBLIOGRAFÍA}

1. Marti A, Martinez J. Adolescent nutrition: the urgent need for acting immediately. An Sist Sanit Navar 2014; 37: 5-8.

2. WHO. Obesity and overweight. Fact Sheet 311 , January 2015. Disponible en: http://www. who.int/mediacentre/factsheets/fs311/en/. Accedido el 09/09/2015.

3. Perez Farinos N. Estrategia NAOS. Estudio ALADINO 2013: Resultados preliminares. Disponible en: http://www.naos.aesan. msssi.gob.es/naos/ficheros/estrategia/ VIII_Convencion/2_ALADINO2013_Convencion_2014_3.pdf. Accedido el 09/09/2015.

4. Osei-Assibey G, Dick S, Macdiarmid J, Semple S, Reilly JJ, Ellaway A et al. The influence of the food environment on overweight and obesity in young children: a systematic review. BMJ Open 2012; 2: 10.

5. Palenzuela Paniagua SM, Perez Milena A, Perula de Torres LA, Fernandez Garcia JA, MaldonaDO Alconada J. Food consumption patterns among adolescents. An Sist Sanit Navar 2014; 37: 47-58.

6. Pablo Rey-Lopez J, Vicente-Rodriguez G, Repasy J, Isabel Mesana M, Ruiz JR, Ortega FB et al. Food and drink intake during television viewing in adolescents: the Healthy Lifestyle in Europe by Nutrition in Adolescence (HELENA) study. Public Health Nutr 2011; 14: 1563-1569.

7. Field AE, Gillman MW, Rosner B, Rockett HR, Colditz GA. Association between fruit and vegetable intake and change in body mass index among a large sample of children and adolescents in the United States. Int J Obes Relat Metab Disord 2003; 27: 821-826.

8. Beunza JJ, Toledo E, Hu FB, Bes Rastrollo M, SeRrano Martinez M, Sanchez Villegas A et al. Adherence to the mediterranean diet, long-term weight change, and incident overweight or obesity the Seguimiento Universidad de $\mathrm{Na}$ varra (SUN) cohort. Am J Clin Nutr 2010; 92 : 1484-1493. 